\title{
USE OF PREPRESS AUTOMATION IN THE CZECH REPUBLIC AND EXAMPLES OF AUTOMATED PROCESSING FOR SELECTED PREPRESS TASKS
}

\author{
Petr Roudný (D), Markéta Držková \\ University of Pardubice, Faculty of Chemical Technology, Department of Graphic Arts and \\ Photophysics, Pardubice, Czech Republic
}

\begin{abstract}
The work is focused on the prepress and possibilities of its automation. The article provides a brief overview of main prepress operations together with software products available for their automation. The information on problematic areas of prepress processing and current use of software tools and prepress automation in the Czech Republic was gathered via the survey with almost a hundred participating companies. The questions about the type of company, the number of its employees and the number of orders received per day were also included so that the results could be better evaluated. As expected, most of the problems in prepress are connected to the printing data received from customers. The reported frequency of different issues and the time needed for the corrections are presented. The results also show that while almost half of the participating companies use a workflow system, which is the main means of automation, often it is not used to the full extent. Further, different possibilities of automated processing were demonstrated for three common issues selected on the basis of the survey results. The tasks included correction of files with default printer marks and a missing bleed, which belong to the most frequent problems, and adding the missing data for processing steps; this issue does not occur so often, but the participating companies reported they spend more time with its solution. The example PDF files were created in Adobe Illustrator and then processed in Adobe Acrobat, Callas pdfToolbox Desktop, Xerox FreeFlow Core, Enfocus PitStop Pro, and Esko Automation Engine. It was verified that when the given software includes the appropriate editing functions and the automated processing is employed, all the tasks can be accomplished very quickly - in the case of the example printing data, it was in less than a minute, usually in a few seconds. Based on the findings, the applicability and benefits of the alternatives considered are outlined. The comparison of functionality of the software tested and a number of their implementations in the participating companies suggests the important role of local resellers and support.
\end{abstract}

Key words: prepress software utilisation, prepress automation, Czech Republic, survey, automation benefits

\section{INTRODUCTION}

Processing of a print job in the prepress stage can be highly automated thanks to full digitalisation of all operations from layout up to imaging or printing. For each step, it is possible to choose from a wide variety of software, and the same applies for automation of the processing in the workflow. On the other hand, the fact that these options are available does not imply their application in the industry or their effective utilisation. This work presents the main findings of a diploma thesis (Roudný, 2020) exploring the current possibilities and use of prepress automation in the Czech Republic.

Today, the prepress workflow is usually based on PDF (Portable Document Format). Figure 1 illustrates the PDF workflow with other processes related to job management and control. The individual steps can be more or less interconnected, depending on the degree of automation, which is commonly accomplished using rule-based approach and job metadata in a suitable format. Besides reducing the need for human intervention and increasing the speed of processing, the automated workflow also helps to ensure proper communication of specifications both between a customer and a company and across all workflow steps, which is the fundamental prerequisite for achieving the required quality in an efficient way. Almost two decades ago, in the study of the former Graphic Arts Technical Foundation (GATF), $88 \%$ of respondents reported they encounter errors in PDF files received from customers (WhatTheyThink, 2002). The authors of a recent survey by the Ghent Workgroup (GWG) see a positive trend regarding the incoming files containing errors, because only $23 \%$ of respondents answered that more than half files contain errors and $46 \%$ reported errors only in a few received files (to $10 \%$ ); however, only $6 \%$ of respondents answered they encounter no errors (Ghent Workgroup, 2018). This indicates there is still room for improvement. 


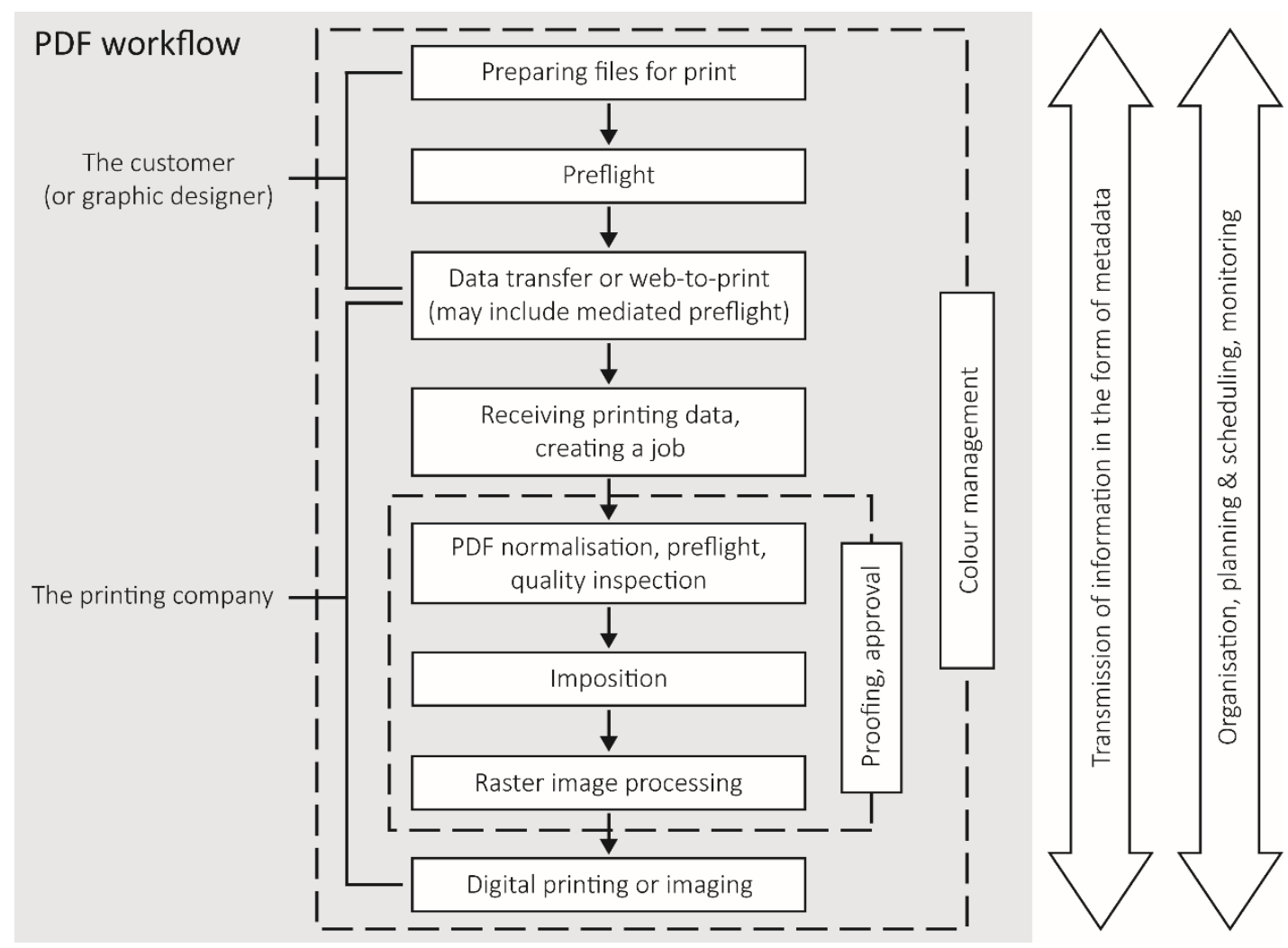

Figure 1: Main steps of PDF workflow and other processes related to job processing before printing

On the customer side, the available prepress solutions helping to produce data conforming to specifications include mainly the preflight tools, either for native files as a built-in feature of a graphic software or using a dedicated plug-in (e.g. Esko DeskPack Packaging Preflight - Esko, 2020a) or application (e.g. Markzware FlightCheck - Markzware, 2020), or for PDF files (e.g. Adobe Acrobat - Adobe, 2020). Data transfer in the next step also can be facilitated by a software, such as Agfa PrintSphere (Agfa, 2018), usually implemented by a printing company. Similarly, it is an integral part of web-to-print solutions (e.g. EFI MarketDirect StoreFront - Electronics for Imaging, 2020a). Enfocus Connect (Enfocus, 2020a) combines data transfer with preflight; more complex solutions further add proofing and approval features (e.g. Kodak InSite Prepress Portal - Kodak, 2018a).

Software for automated preflight and editing of received PDF files used by printing companies ranges from Adobe Acrobat (Adobe, 2020), to applications as Enfocus PitStop Pro (Enfocus, 2020b) and Callas pdfToolbox (Callas software, 2020), up to the tools included in complex workflow systems, usually with built-in Enfocus PitStop or Callas software technology. Some workflow systems offer also their own technology, such as EFI Fiery Preflight (Electronics for Imaging, 2020b), Heidelberg Preflighter (Heidelberg, 2012) and OneVision Asura (OneVision Software, 2020). Further capabilities include the raster preview of separations (e.g. Agfa Apogee - Agfa, 2020), advanced comparison of PDF files (e.g. Kodak Prinergy Workflow - Kodak, 2018b) and other quality control features (e.g. Esko Automation Engine, Esko, 2020b). The 2020 release of Enfocus PitStop employs computer vision techniques to check the visual content of a PDF file (Enfocus, 2020c). Among the software for imposition, automation is supported e.g. by Tilia Labs Phoenix (Tilia Labs, 2019). The final steps, i.e. raster image processing and imaging or digital printing, can be a part of the automated workflow as well. Appropriate colour management features are important across the whole process, which is reflected in all prepress workflow systems and applications.

This outline has listed just a few examples of products available on the market; a comprehensive (but still not exhaustive) overview is in Roudný (2020). The next part of this work presents the survey conducted among printing companies to gain an insight into utilisation of these tools in the industry and identify problematic areas of prepress automation in the Czech Republic. Based on the results, missing data for processing steps (namely varnishing and cutting), problems with bleed and problems with printer marks (crop and registration marks, colour bars, etc.), were chosen as model situations. The last part presents the corresponding source data and example solutions in Adobe Acrobat, Callas pdfToolbox, Enfocus PitStop, Xerox FreeFlow Core, and Esko Automation Engine. 


\section{SURVEY ON PREPRESS AUTOMATION IN THE CZECH REPUBLIC}

The survey among printing companies in the Czech Republic employed an online questionnaire. The introductory questions assessed general information about the involved companies (main type of production, number of employees and average number of jobs per day) to provide a context for evaluation of answers concerning the prepress practices and issues, as well as the software, specifications and standards used by the companies.

\subsection{Survey participants}

The questionnaire was completed by 94 companies from altogether 426 companies randomly selected across all regions of the Czech Republic based on the annually published directory (ISMC, 2018) and information available on the internet.

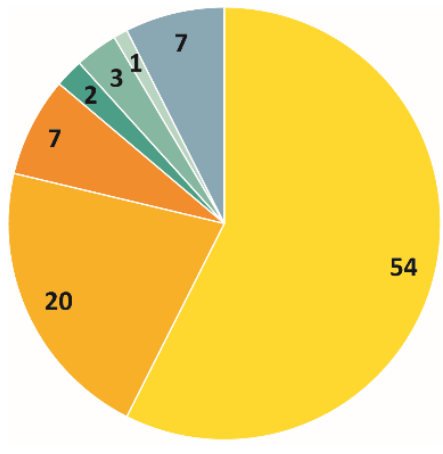

54

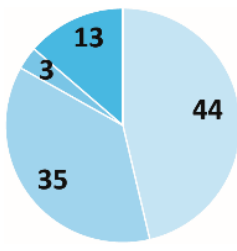

$1-10$

$11-50$

$51-100$

101 and more

(n)
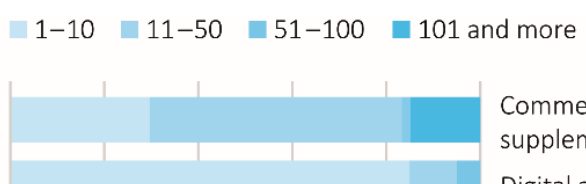

Commercial offset printing (possibly supplemented by digital or other printing)

Digital and/or large format printing

Label and/or packaging printing

Magazine and/or newspaper printing

Textile printing and/or promotional products printing

Publishing

Other

b)

c)

Figure 2: Survey responses to the questions on a) the main type of production and b) the number of employees, with c) percentage representation of the latter according to the main type of production

Figures 2 and 3 show characteristics of the participating companies. Regarding the main type of production, the companies who answered "Other" specified letterpress printing and die-cutting, manufacture of paper packaging, signmaking and orientation systems, screen printing and related services, and printing on digital media; one company combines more sectors. As there is no current and detailed official statistics on the structure of the Czech printing industry available, it is not possible to classify the sample of respondents as representative or not. However, taking into account the production and size of the involved companies, the sample can be considered satisfactory. When looking at the number of employees and jobs per day, the overall pattern in the graphs is similar, with the ratio of jobs and employees higher for digital and large format printers and smaller for large commercial offset, label, packaging, magazine and newspaper printers. 


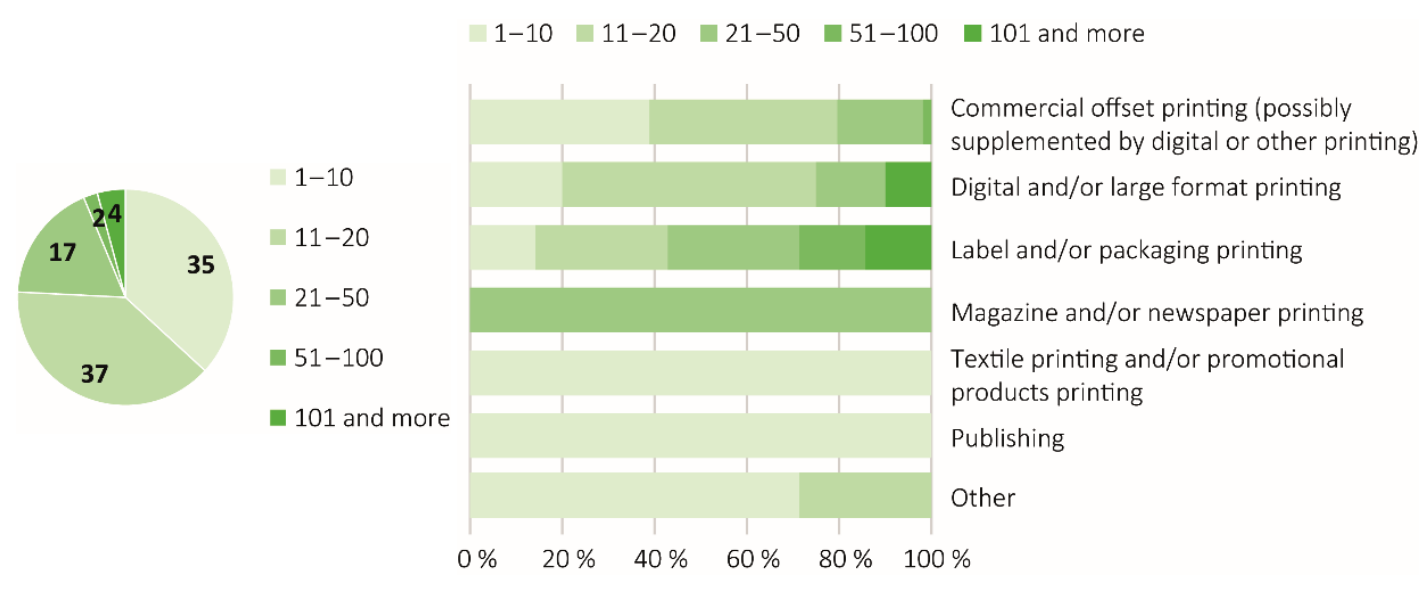

a)

b)

Figure 3: a) Survey responses to the question on the number of jobs per day, with b) their percentage representation according to the main type of production

\subsection{Prepress practices and issues}

The main part of the questionnaire, focused on prepress, started with the question whether and how companies support graphic designers or customers in preparing printing data. Only 6 companies do not provide any support; among the remaining 88 respondents, 50 provide at least some kind of support and the other combine more options. About 80 \% (76 companies) explain their requirements on request, or when it is necessary to correct the received data. But more than a half of them, 40 companies, do not use any other option. Only 29 companies have the requirements regarding printing data specified on their website, and just 4 companies offer free download of preflight profiles, ICC profiles, droplets, etc. In 16 companies, printing data are automatically checked when transferred to the company. With a few exceptions, the results clearly show the overall lack of attention paid to this area, especially when considering the answers to the following question showing that almost all respondents encounter problems with poorly prepared printing data and also the other common problems are related to the communication with clients (Figure 4). When examining how frequently the companies encounter jobs with incorrect printing data, almost half of the respondents reported that it is the case of approx. each third job; for about one third of respondents, it even makes a half of jobs or more. These numbers are higher than reported in the GWG survey participated by 1109 respondents worldwide (Ghent Workgroup, 2018).

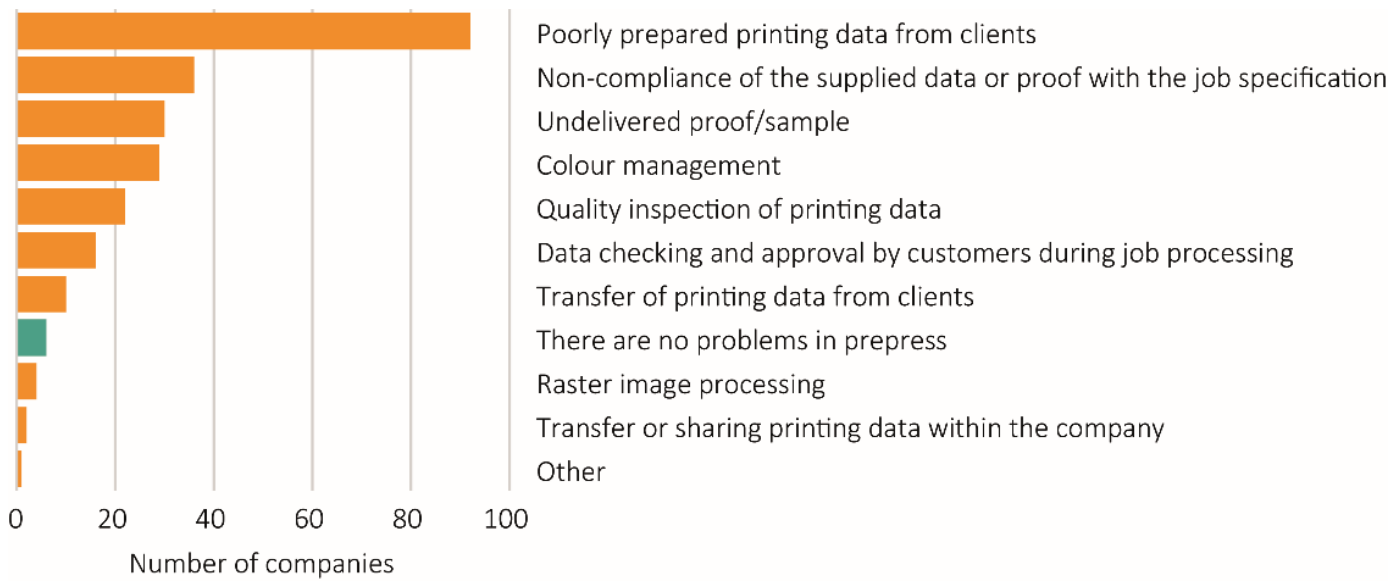

Figure 4: Survey responses to the question in which areas of prepress are encountered problems

Two questions explored the frequency of selected issues in the received printing data and the time typically needed to solve them; the results are presented in Figure 5. The most common problems are bleed issues and wrong resolution or format of raster images. Almost all companies also encounter the problems with $\mathrm{PDF} / \mathrm{X}$ compliance, colour space, fonts and page format, at least occasionally. When analysing how time- 
consuming is to solve individual issues, on average, the highest number of respondents can solve the problems in 1-10 minutes (45\%) and about $20 \%$ need less than 1 minute. On the other hand, about $25 \%$ need 11-59 minutes and $10 \%$ of respondents even more than an hour. Among the individual categories, more time-consuming is to deal with the missing data for processing steps and problems with transparency or its flattening; the largest delays are caused when the printing data do not match with the delivered proof, PDF files cannot be opened or processed, and raster images have wrong resolution or format.

The rather high ratio of answers that indicate solving problems within 1 or 10 minutes suggests the automated or semi-automated processing. However, 53 respondents (56 \%) answered the optional open question allowing to provide more details about the way the most common issues are solved, where 30 respondents reported that they return the data to the customer or graphic designer. It was found out that the majority of these respondents indicated a short time needed to deal with the issues. Therefore, in these companies, the real time needed to completely solve the problems is probably longer than they reported. Only two respondents reported making corrections in an automated workflow.

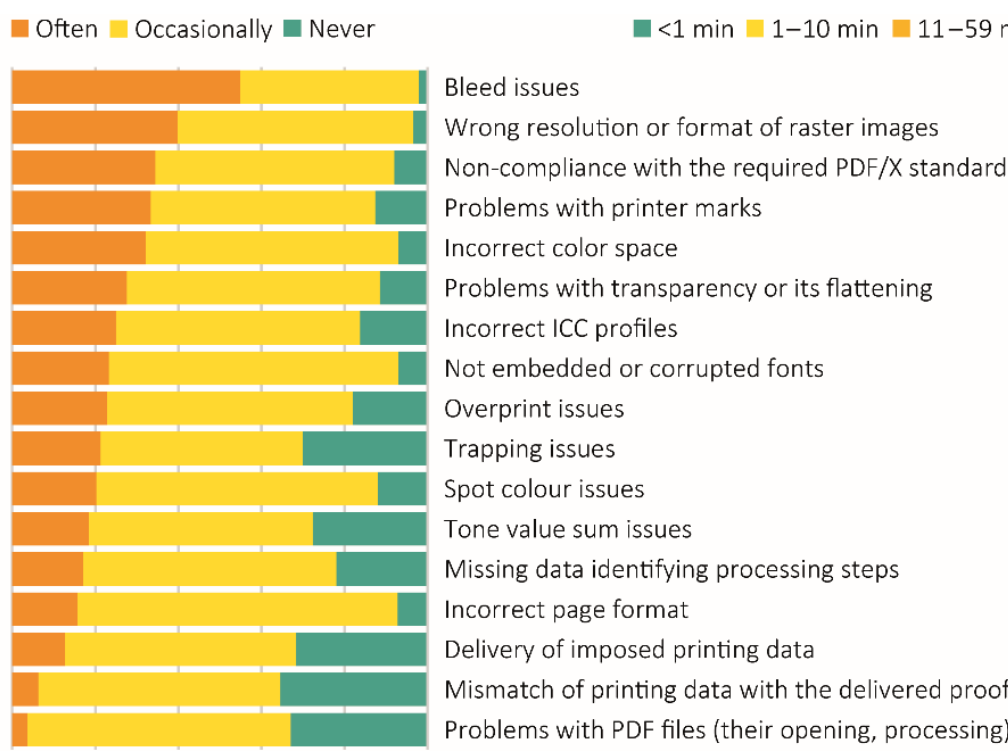

$0 \% \quad 20 \% \quad 40 \% \quad 60 \% \quad 80 \% \quad 100 \%$

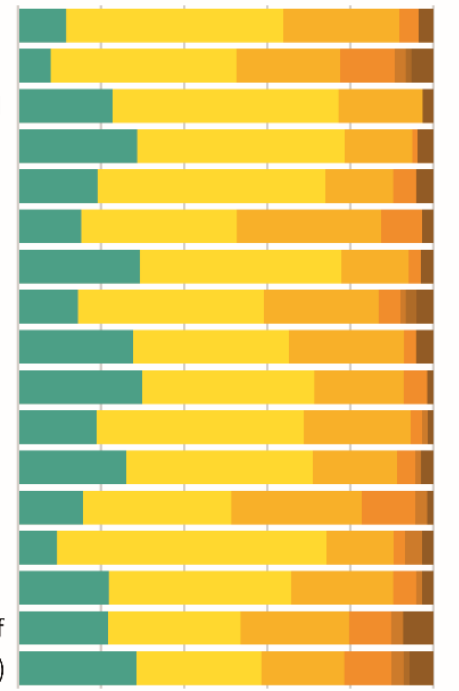

$0 \% \quad 20 \% \quad 40 \% \quad 60 \% \quad 80 \% \quad 100 \%$

Figure 5: Percentage representation of survey responses specifying how often are encountered particular issues in the received printing data (left part) and how long does it typically take to solve these issues (right part)

Another question was how much time does it approximately take to check and process a correctly prepared printing PDF (from its receiving up to raster image processing). Almost two thirds of respondents answered it takes 1-10 minutes and about one third 11-59 minutes; only a few respondents need longer time. These results confirm that the low efficiency in prepress is mostly caused by poorly prepared printing data that need correction, which is often due to the lack of communication between a company and a customer.

\subsection{Prepress software}

The following questions asked about the software solutions used in the companies for prepress operations and their automation.

Concerning the prepress workflow systems, above one third of respondents use only independent applications and modules from different manufacturers, not communicating with each other. About $17 \%$ combine the software from different manufacturers into the workflow and $5 \%$ use the custom-programmed workflow system. The remaining companies listed Caldera Nexio, Enfocus Switch, Esko Automation Engine, Kodak Prinergy, Heidelberg Prinect, Agfa Apogee, Asanti or Arkitex, Fujifilm XMF, Efi Fiery Workflow Suite, OneVision Software solutions, and Xerox FreeFlow Core. Among these, Heidelberg Prinect used by $12 \%$ of respondents is the most common, while the other systems are used by $5 \%$ or less.

Among PDF preflight and editing software, the most commonly used is Adobe Acrobat (53\% of respondents) and Enfocus PitStop Pro (40\%); $14 \%$ use the built-in preflight in a workflow system and only a few respondents use Enfocus PitStop Server or Callas pdfToolbox Desktop. Almost a quarter of respondents check the data only visually and $4 \%$ do not preflight nor correct data. 
When examining software coverage of other prepress areas, about two thirds of respondents use software for raster image processing and imposition and about one third for job management, colour management, job monitoring in production and information system functions. Further, $21 \%$ use software for certified preview or proofing in a company, $16 \%$ for data transfer from a customer, $10 \%$ for approval workflow or certified online soft proofing, and $4 \%$ for mediated automatic preflight on the side of a customer. In general, these numbers are low.

Concerning the ways of simplifying routine work in prepress, predefined actions and batch processing are used by $40 \%$, hot folders by $28 \%$ and JDF (Job Definition Format) by $10 \%$ of respondents, at least; $17 \%$ of respondents answered that they do not know the particular way, because all automated tasks are done by some application or workflow system as set up since implementation. Some respondents noted in the answer other ways, such as definition of keyboard shortcuts, use of templates, plug-ins, appropriate settings, etc. More than a quarter of companies do not use any of these options.

Considering these results, on the one hand, the responses suggest quite satisfying situation among printing companies as almost every second one uses a prepress workflow system and about three quarters automate at least some operations. On the other hand, the high ratio of incorrect files received from customers, the time needed to solve these issues and the low number of companies using automated preflight and other options show that the prepress workflow systems could be utilised more efficiently where available and more automation tools should be implemented overall. At the same time, it is important to stress that the investment into any automation needs to be carefully considered and, even then, it may require further effort to make a full use of it. For example, one company that offers mediated preflight and data upload using Enfocus Connect reported that customers are not interested in this option, in spite of the marketing campaign.

\subsection{PDF standards and specifications}

According to the answers, almost every respondent is familiar with PDF/X standards (96\%), while PDFXready (17\%) and GWG (10\%) specifications are much less known among respondents. This explains the overall weak support provided to graphic designers or customers in preparing printing data. It can be expected that companies will achieve a substantial decrease in error rate for printing data they receive if their knowledge in this area improves.

When analysing the particular PDF standards and specifications used in the involved companies, the most respondents (59\%) use PDF/X-1a:2001. This corresponds with the recent information that it is still the most widespread PDF/X standard in the Czech Republic (Lozan, 2017). The less restricted standard, PDF/X-4, is used by $38 \%$ of companies, often together with PDF/X-1a:2001. Only several respondents use PDFX-ready V1.3, PDFX-ready V2, or GWG2015 specifications. However, about a third of respondents use their own customised settings. About $10 \%$ of respondents do not use any standard or specification or do not know. The former answered that they print whatever the customer sends, without any changes, i.e. with no control of the input data quality. This approach was reported also by some companies from those who otherwise use the standardised settings but leave the responsibility on the customer (15\% of respondents).

\subsection{Further remarks}

The evaluation of the survey was quite complicated due to the inconsistent use of terms, although the questionnaire was in the Czech language. The answers show that some respondents misunderstand or confuse the concepts as preflight, prepress workflow system, PDF standards and specifications, listing the software or formats without the corresponding features. Naturally, when the awareness of the current technology and possibilities is low, it is difficult to achieve any progress or at least to do it efficiently. Besides the education on the side of printing companies, higher expertise of graphic designers would improve the situation as well, since the understanding of the issue can facilitate a large amount of work. As shown in the study of Liao and Lü (2017), which investigated the possibilities to optimise the preflight settings, the complexity of preflight should be adjusted according to the customer situation. The comprehensive preflight settings are recommended only when a graphic designer on a customer side is very well acquainted with the exact requirements of the printing company. However, at least the essential parameters such as document size, transparency, bleed and resolution should be checked in any case. 


\section{AUTOMATED PROCESSING OF MODEL PREPRESS TASKS}

This part deals with the processing of three model tasks to practically examine the current possibilities of prepress automation. The tasks were selected according to the findings from the survey. The printing data with default printer marks and a missing bleed represent the most frequently encountered problems, while the issue with the missing data for processing steps requires more time to be solved (see Figure 5). The example printing data were created in Adobe Illustrator and saved to PDF. One model job is a bookmark (Figure 6), where partial varnishing of the illustration of screen printing is expected. The illustration was also used separately as the second model job, which is expected to be cut after printing, for example in the form of a sticker (Figure 7). The illustration consists of many objects to make its automatic selection more difficult. The default printer marks were deliberately added to the printing data for both jobs; in addition, a second version without bleed and printer marks was created for the bookmark. All objects are vectorbased by default, but versions with rasterised objects were also created and used during testing.

The first aim was to remove the default printer marks and add the required ones. The second aim was to add a bleed when missing. The third aim was to create a new design element either in a spot colour or in a new layer or both and set it to overprint. In the case of the bookmark, it is the filled element in the shape of the illustration to be varnished. In the case of the illustration alone, it is a line around the motif to be cut during finishing. The example PDF files were processed in Adobe Acrobat X Pro, Callas pdfToolbox Desktop 11, Enfocus PitStop Pro 2020, Xerox FreeFlow Core 5.4.1, and Esko Automation Engine 18.1.0. A detailed description of all procedures and settings is in Roudný (2020).

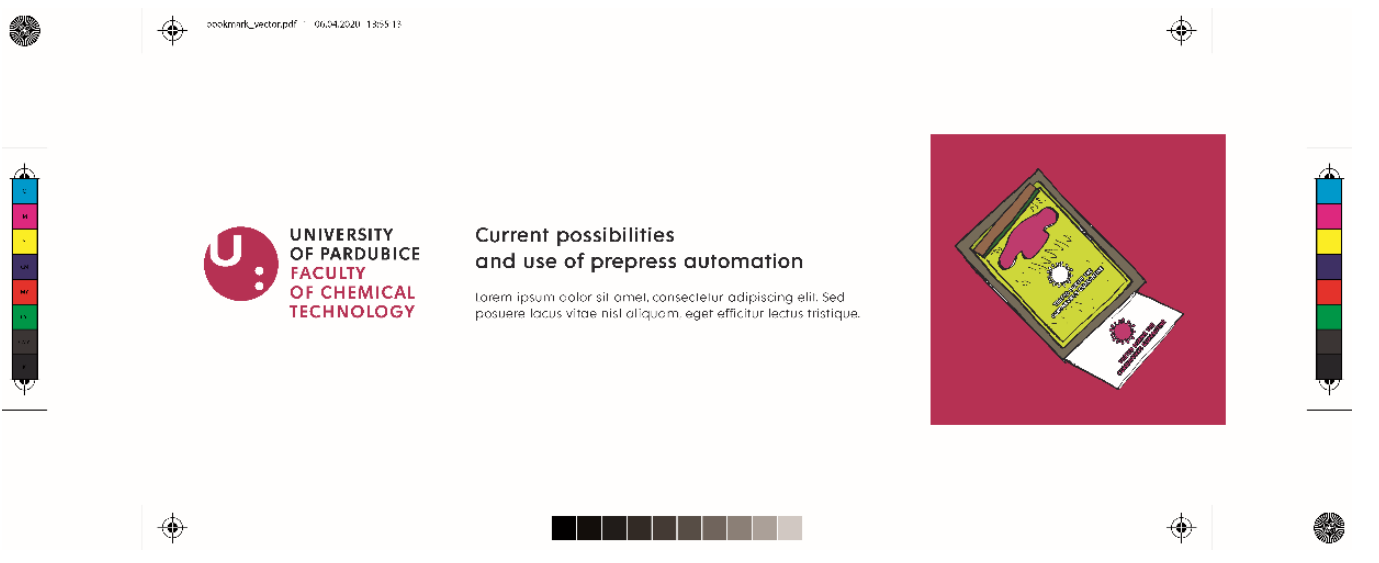

Figure 6: A preview of the first printing data created for testing - the bookmark with the default printer marks and without the data for partial varnishing (scale 1:2); the second printing data comprise the same content but cropped to the TrimBox size (without the printer marks and bleed)

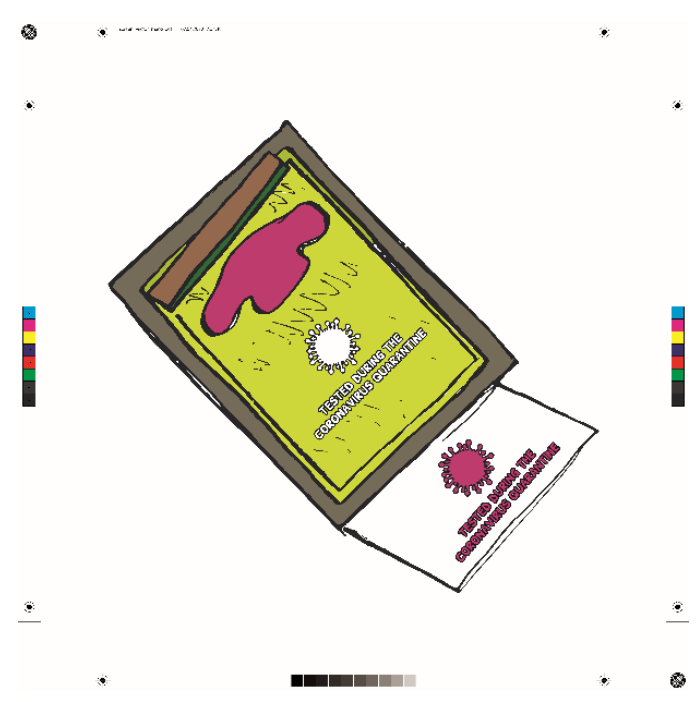

Figure 7: A preview of the third printing data created for testing - the illustration of screen printing with the default printer marks and without the data for cutting (scale 1:4) 


\subsection{Adobe Acrobat}

Adobe Acrobat enables automated processing of PDF files using actions to perform the required operations, including those defined in preflight profiles with appropriate checks and fixups. When the action is executed, all operations run in the background. It is possible to batch-process more files at once. Considering the selected model tasks, this way can be fixed only the issue with the printer marks. The corresponding action involved defining a file or folder with files that should be processed, removing the content outside the BleedBox, adding the correct printer marks, if required, setting the proper CropBox size, and saving the resulting file(s). In some cases, the file with the missing bleed can be also corrected automatically, but only when the content of the bleed area is present in the PDF file and just hidden due to the CropBox size set to that of the TrimBox. Creating or editing objects in the case of missing bleed and technical elements for varnishing or cutting requires manual editing and still the possibilities are very limited.

\subsection{Callas pdfToolbox Desktop and Enfocus PitStop Pro}

The desktop versions of Callas pdfToolbox and Enfocus PitStop Pro offer advanced options for PDF file editing and allow automated processing of all three tested tasks. In general, more approaches can be used to accomplish the given task in both of them. The optimal procedure is derived from the complexity and other features of the particular design and often can be chosen on the basis of the suitable checks.

In Callas pdfToolbox, fixups and checks can be combined into profiles and further into process plans, so that all the required tasks can be completed at once. As in Adobe Acrobat, they can be applied in batch processing. Moreover, the values of parameters can be defined as variables and later, during execution, individually specified by the operator according to the given job. The steps for correcting the printer marks were the same as in Adobe Acrobat. The methods available for adding the missing bleed without changing the content of the TrimBox are "Mirror as images (edges and corners)", "Repeat the last pixel as image" and "Mirror page objects". Only the last one preserves the vector objects in bleed and therefore it was used in the testing; in other cases, the created bleed is a raster image. Another option is to use a fixup "Generate bleed by upscaling". To create the element for varnishing or cutting, the procedure was based on a fixup "Create and apply shapes". It was verified that the selection of the appropriate object(s) can be based on a certain number of nodes in the path or size of the object. For complex illustrations, it might be necessary to choose the options "From tracing page content (including white areas)" (or, on the contrary, excluding white areas), "Render only outside shape" and "Reduce shape to outer borders". It was also possible to create the shape from a defined layer. The size of the resulting shape could be adjusted to account for the manufacturing tolerances. Finally, the created shape was applied as a filled object for varnishing or a stroked object for cutting, with defined properties. Figure 8 shows the example of the resulting data with the path for cutting. In addition, it was possible to define a more complex workflows within the process plans, including not only the described modifications, but also the imposition of both the printed image and the varnishing or cutting element into two separate PDF files.

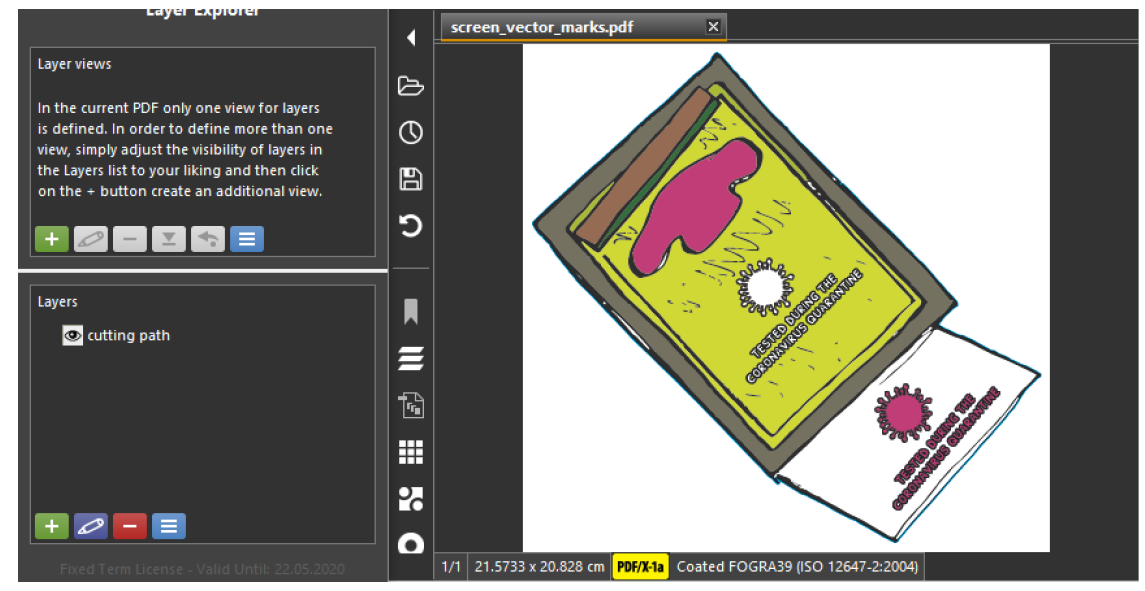

Figure 8: Modified printing data (see Figure 7) with the created layer "cutting path" containing the stroked object in a spot colour set to overprint, in Callas pdfToolbox Desktop 11

In Enfocus PitStop Pro, a plugin for Adobe Acrobat, the automated processing can employ preflight profiles, global changes and action lists, where also the manually performed and recorded operations can be used. 
These all can be combined into the so-called QuickRuns. There is also the possibility to use the variables and logical operators. For correcting the printer marks, the procedure is again similar as in the previous two applications, but in PitStop it is not possible to define the added marks in detail; only the InDesign style (which interferes with the BleedBox) or the QuarkXpress style can be chosen. The missing bleed can be fixed by mirroring or enlarging the content at the TrimBox edges; when generating the bleed in PitStop, the type of data (vector or raster) is preserved. Several options were tested to create the element for varnishing or cutting. For vector objects, it is always based on combining and dividing shapes; in this particular case, the "Unite line art" action was used after selecting the objects by size, with the option to "Keep original objects" to create the new shape. If the resulting shape shows any undesired features (such as occasional holes) that can arise when editing complex illustrations, the action can be used repeatedly. Then, the fill or stroke and overprint properties of the object were defined. As in Callas pdfToolbox, it was also possible to adjust the size and create a dedicated layer. The procedure could be further enhanced by employing the "Select overlapping objects" action. For some designs, the action "Select line art by total number of nodes" may be more appropriate. In the case of raster images, the action "Trace the selected object(s)" with the option to "Ignore white holes" was applied to the selection made using the "Select by image size" action. As an alternative, "Select objects inside region" was used, which can be applied also for vector objects.

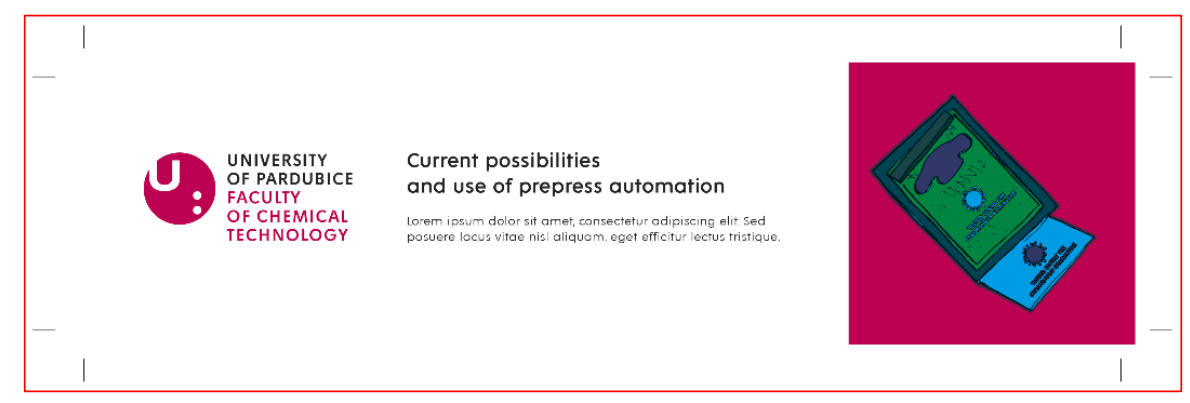

Figure 9: A preview of the printing data (see Figure 6) modified using Enfocus PitStop Pro 2020, with the corrected printer marks and the filled object in a spot colour set to overprint defining the area for partial varnishing (scale 1:2); the CropBox is marked with a red line

Each of these software solutions has some advantages in terms of possibilities, quality, and speed of processing; the comparison of time needed to create the cutting path in dependence on the type of objects is given in Table 1. Therefore, their suitability must be evaluated in the context of particular requirements. While Callas pdfToolbox Desktop offers batch processing, process plans and imposition and thus appears to be more powerful in terms of automation, Enfocus PitStop Pro offers the option of manual editing that can be recorded to Action Lists, which can be indispensable in some cases. In general, the range of functions in Callas pdfToolbox and Enfocus PitStop Pro is still being upgraded.

Table 1: Speed of data processing when creating the cutting path in Callas pdfToolbox Desktop 11 and Enfocus PitStop Pro 2020 (for the data see Figure 7); raster procedure means the use of "Raster selection" and "Add trace path" actions in Enfocus PitStop Pro and "Create shape from tracing page content" in fixup in Callas pdfToolbox Desktop

\begin{tabular}{llll}
\hline Data type & Callas pdfToolbox Desktop & Enfocus PitStop Pro & Comparison \\
\hline Vector data & $1.7 \pm 0.2 \mathrm{~s}$ & $0.9 \pm 0 \mathrm{~s}$ & $\begin{array}{l}\text { Enfocus PitStop Pro } \\
1.9 \text { times faster }\end{array}$ \\
$\begin{array}{l}\text { Vector data, raster procedure } \\
\text { of creating the cutting path }\end{array}$ & $1.5 \pm 0.2 \mathrm{~s}$ & $4.3 \pm 0.2 \mathrm{~s}$ & $\begin{array}{l}\text { Callas pdfToolbox Desktop } \\
2.9 \text { times faster }\end{array}$ \\
Raster data & $5.6 \pm 0.1 \mathrm{~s}$ & $24.6 \pm 0.3 \mathrm{~s}$ & $\begin{array}{l}\text { Callas pdfToolbox Desktop } \\
4.4 \text { times faster }\end{array}$ \\
\hline
\end{tabular}

\subsection{Xerox FreeFlow Core and Esko Automation Engine}

From the two prepress workflow systems tested, Xerox FreeFlow Core includes the built-in Callas pdfToolbox technology; the checks, fixups, preflight profiles, and process plans created in Callas pdfToolbox Desktop can be loaded and used in Xerox FreeFlow Core, but it is not possible to define them directly in Xerox FreeFlow Core. Xerox FreeFlow Core 5.4.1, in particular, has a built-in Callas pdfToolbox 10.2, while the Desktop application was used in version 11; nevertheless, all functions utilised in this work (see 3.2) are available 
also in version 10.2 and thus all the automated procedures defined in Callas pdfToolbox Desktop 11 could be used in the workflow system. When employing the additional features available in Xerox FreeFlow Core and creating more advanced workflow, including e.g. the PDF file optimisation, it was possible to save further work and time compared to batch processing in Callas pdfToolbox Desktop or Adobe Acrobat.

The second prepress workflow system, Esko Automation Engine, offers the most automation features among the software solutions used for testing. However, as its version 18.1.0 has a built-in Enfocus PitStop 2018 and the action lists were prepared in Enfocus PitStop Pro 2020, some features, including the "Add tracing path" action, were missing and only the action lists with supported functions could be imported and used within Esko Automation Engine. On the other hand, it is possible to create user-defined action lists directly in Esko Automation Engine; the Enfocus PitStop licence is not needed. As in Xerox FreeFlow Core, all three model tasks described above were successfully completed in Esko Automation Engine, together with the additional operations included in the more complex workflow, such as the optimisation, imposition and separate processing of the printed image and the varnishing or cutting element.

In general, the workflow systems as such offer the greatest possibilities for the automation of the entire job processing and the efficient use of the metadata available for a particular job. However, it is necessary to take into account that the new versions of the built-in technology are always implemented with some delay. Therefore, in this respect, the available options are limited compared to the latest version of the respective standalone applications.

\section{CONCLUSIONS}

This work helped to gain insight into the current situation in prepress automation in the Czech Republic. Based on the results of the survey among printing industry participants and practical demonstration of possible solutions of the three model prepress tasks, it can be concluded that the time needed to deal with the commonly encountered issues can be significantly reduced through automation using the existing software. The software based on either the Callas pdfToolbox or Enfocus PitStop technology could automatically complete all prepress operations in question. In Adobe Acrobat, the range of functions is more limited and some operations could not be done without the use of an additional software, but still it enables automated batch processing of many tasks. The survey shows that, overall, the awareness of the available options is rather low, which indicates a large room for improvement being possible even without a substantial investment if the companies learn about the automation possibilities in more detail and, based on the process analysis, implement the appropriate solution. On the other hand, some companies are well informed about the current technology and options in prepress and benefit from its automation. Further, it was observed that although Callas pdfToolbox Desktop and Enfocus PitStop Pro are applications with the same purpose and largely with a similar functionality, much higher use of the Enfocus PitStop Pro can be seen among the respondents. This may be because there is no Callas pdfToolbox Desktop reseller on the Czech market, which suggests the important role of local business and technical support.

\section{ACKNOWLEDGMENTS}

The authors thank all respondents for taking part in the survey, Martin Šaněk and Nextar Group for the access to Xerox Free Flow Core, Dominik Štýbr and OTK GROUP for the access to Esko Automation Engine, and Four Pees for the possibility to use Callas pdfToolbox.

\section{REFERENCES}

[1] Adobe: "Acrobat User Guide - Preflight (Acrobat Pro)", URL: https://helpx.adobe.com/acrobat/userguide.html?topic=/acrobat/morehelp/preflight_acrobat_pro_.ug.js (last request: 2020-09-10).

[2] Agfa: "PrintSphere", URL: https://www.agfa.com/printing/wpcontent/uploads/sites/19/2019/09/printsphere-brochure-en-web.pdf (last request: 2020-09-10).

[3] Agfa: "Apogee", URL: https://www.agfa.com/printing/products/apogee/ (last request: 2020-09-10).

[4] Callas software: "pdfToolbox", URL: https://www.callassoftware.com/en/products/pdftoolbox (last request: 2020-09-10).

[5] Electronics for Imaging: "EFI MarketDirect StoreFront", URL: https://www.efi.com/products/productivity-software/efi-marketdirect-customer-engagementplatform/efi-marketdirect-storefront/overview/ (last request: 2020-09-10). 
[6] Electronics for Imaging: "Fiery JobFlow", URL: https://www.efi.com/products/fiery-servers-andsoftware/fiery-workflow-suite/fiery-jobflow/overview/ (last request: 2020-09-10).

[7] Enfocus: "Connect ALL: PDF quality control for job submission", URL: https://www.enfocus.com/en/connect-all (last request: 2020-09-10).

[8] Enfocus: "PitStop Pro: PDF checked, fixed, ready", URL: https://www.enfocus.com/en/pitstop-pro (last request: 2020-09-10).

[9] Enfocus: "PitStop 2020 Released", URL: https://www.enfocus.com/en/news/pitstop-2020-released (last request: 2020-09-10).

[10] Esko: "DeskPack Packaging Preflight for Adobe Illustrator", URL: https://www.esko.com/en/products/deskpack/plugins/packaging-preflight (last request: 2020-0910).

[11] Esko: "Automation Engine", URL: https://www.esko.com/en/products/automation-engine/features (last request: 2020-09-10).

[12] Ghent Workgroup: "GWG 2017 PDF Survey Results", URL: https://www.gwg.org/gwg-2017-pdfsurvey-results/ (last request: 2020-09-10).

[13] Heidelberg: "Prinect Prepress Manager", URL: https://www.heidelberg.com/global/media/en/global_media/products ect_prepress_manager_product_guide.pdf

[14] ISMC: "Polygrafie České republiky 2018 - Kompletní seznam firem z oblasti polygrafie, souvisejících činností a jejich dodavatelü" [2018 Graphic Arts Industry of the Czech Republic - Complete directory of companies from the printing industry and related fields and their suppliers], 23rd edition, (ISMC Bohemia, Brno, 2018).

[15] Kodak: "InSite Prepress Portal", URL: https://www.kodak.com/uploadedFiles/PinergyWorkflow/Insite_Prepress_Portal/InSitePrepressPortalSellSheet.pdf (last request: 2020-09-10).

[16] Kodak: "Prinergy Workflow", URL: https://www.kodak.com/uploadedFiles/PinergyWorkflow/Pinergy-Workflow/PrinergySellSheet.pdf (last request: 2020-09-10).

[17] Liao, Y., Lü, X.: "Optimized three-stage strategy of orderly management of file stream in network printing workflow", Journal of Systems Science and Information 5 (1), 74-87 (2017). doi:10.21078/JSSI-2017-074-14.

[18] Lozan, P.: "Odkládáte implementaci PDF/X-4 na neurčito? Stephan Jaeggi: Je to jen výmluva" [Are you postponing the implementation of PDF/X-4 indefinitely? Stephan Jaeggi: It's just an excuse], URL: http://www.pdf-x.cz/stephan-jaeggi-rozhovor-je-to-jen-vymluva/ (last request: 2020-09-10).

[19] Markzware: "FlightCheck (Preflight for Print)", URL: https://markzware.com/products/flightcheck/ (last request: 2020-09-10).

[20] OneVision Software: "Asura", URL: https://www.onevision.com/solutions/prepress-software/asura/ (last request: 2020-09-10).

[21] Roudný, P.: "Current possibilities and use of prepress automation", MSc thesis, University of Pardubice, Pardubice, 2020. URL:

https://portal.upce.cz/StagPortletsJSR168/CleanUrl?urlid=prohlizeni-prace-detail\&praceldno=37988 (last request: 2020-09-10).

[22] Tilia Labs: "Automation and Integration", URL: https://www.tilialabs.com/phoenix/automation/ (last request: 2020-09-10).

[23] WhatTheyThink: "GATF Studies the Reality of PDF, Top Five Benefits", URL: http://whattheythink.com/news/17122-gatf-studies-reality-pdf-top-five-benefits/ (last request: 2020-09-10).

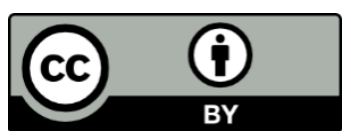

(C) 2020 Authors. Published by the University of Novi Sad, Faculty of Technical Sciences, Department of Graphic Engineering and Design. This article is an open access article distributed under the terms and conditions of the Creative Commons Attribution license 3.0 Serbia (http://creativecommons.org/licenses/by/3.0/rs/). 